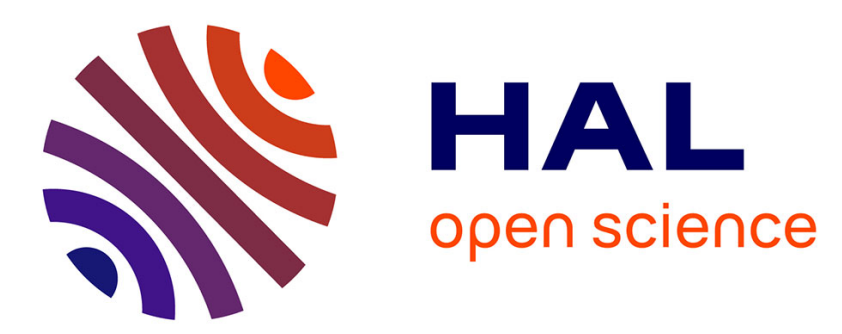

\title{
Impacts territoriaux et compensations socio-environnementales
}

Julie Gobert

\section{To cite this version:}

Julie Gobert. Impacts territoriaux et compensations socio-environnementales. L'énergie à découvert, 2013, 978-2-271-07678-6. hal-01841231

\section{HAL Id: hal-01841231 \\ https://hal-enpc.archives-ouvertes.fr/hal-01841231}

Submitted on 30 Jul 2018

HAL is a multi-disciplinary open access archive for the deposit and dissemination of scientific research documents, whether they are published or not. The documents may come from teaching and research institutions in France or abroad, or from public or private research centers.
L'archive ouverte pluridisciplinaire HAL, est destinée au dépôt et à la diffusion de documents scientifiques de niveau recherche, publiés ou non, émanant des établissements d'enseignement et de recherche français ou étrangers, des laboratoires publics ou privés. 


\subsection{Impacts territoriaux et compensations socio-environnementales}

\section{Julie Gobert}

Urbaniste - Politiste - Jeune chercheure à I'Université Technologique de Troyes - Centre de Recherches et d'Etudes Interdisciplinaires sur le Développement Durable - julie.gobert@gmail.com Mots-clés : justice distributive, justice procédurale, compensation socio-environnementale

Les infrastructures de production (infrastructures ponctuelles comme les centrales hydro-électriques ou les bioraffineries) et de transport d'énergie (infrastructures linéaires comme les lignes électriques) créent sur leur territoire d'accueil de multiples impacts négatifs et positifs, qui pèsent dans leur acceptabilité sociale.

\section{Prendre en compte les impacts négatifs et positifs d'une infrastructure}

Ainsi, les effets négatifs sur l'environnement (ennoiement d'espaces ruraux ou forestiers pour créer un lac de rétention en amont d'un barrage; coupure paysagère due à une ligne électrique; nuisances, risques et pollutions possibles sur l'environnement proche...) se concentrent souvent sur un espace circonscrit, celui à proximité immédiate de l'infrastructure, sauf dans le cas de pollution atmosphérique (centrale thermique au charbon par exemple) ou d'accidents majeurs lorsque des rejets dans le sol, l'air ou l'eau se dispersent bien au-delà du territoire d'impact « habituel ».

Les effets positifs eux sont plus diffus et concernent à la fois le territoire d'implantation, la région, voire des territoires plus vastes. Ainsi l'énergie produite à un endroit tend à être largement redistribuée, les riverains n'étant pas nécessairement les usagers du produit final. Certes, le territoire d'accueil perçoit des retombées positives (fourniture d'énergie, développement économique, création ou maintien d'emplois sur la zone, etc.), mais souvent sans que ces retombées puissent être considérées comme une contrepartie juste et probante aux externalités négatives. Ce déséquilibre peut engendrer un sentiment d'injustice qui se concrétise souvent par une opposition forte et structurée.

Les opposants, qu'ils soient riverains ou non, sont en capacité de retarder la mise en chantier de l'équipement, s'ils intentent une action judiciaire, mobilisent des acteurs politiques majeurs pour freiner la procédure, etc. Plusieurs facteurs viennent expliquer cette forte réactivité aux ouvrages de fourniture et de distribution d'énergie:

- Une conscientisation environnementale plus forte de la population en général, même si selon la catégorie socio-professionnelle, l'âge, le territoire d'appartenance (l'ancrage à ce 
territoire, la manière de l'appréhender), des différences importantes de ressenti sont observées ;

- Une structuration conséquente des acteurs se présentant comme les défenseurs de leur cadre de vie, de leur milieu et de leur environnement. Ils ont progressivement acquis de plus en plus de ressources et de compétences (sociales, financières, expertise) et sont en capacité d'investir différentes arènes (débat public, tribunal, etc.).

Or les arènes institutionnelles de concertation répondent rarement à toutes les questions et problématiques que pose ou révèle le projet aux riverains. Malgré les études d'impact, les enquêtes publiques, voire selon certains projets le débat public, des angles morts existent et un ressentiment peut naître chez des habitants qui considèrent n'avoir pas été sollicités en amont du projet ou n'avoir été que consultés, sans être pris pour des interlocuteurs de premier plan.

\section{Compensations socio-environnementales}

Au gré d'un apprentissage qui se rôde au fur et à mesure des projets, les maîtres d'ouvrage (transporteurs et producteurs d'énergie) ont dû renouveler leur manière d'agir ; ils ont conscience que l'intérêt général n'est plus une justification suffisante pour légitimer leur projet. Celui-ci doit s'accommoder des multiples intérêts locaux. Aussi faut-il faire appel à une meilleure justice procédurale (apprendre à connaître le territoire et les populations qui y vivent, leurs perceptions, leurs attentes, leurs besoins; mettre en place une stratégie participative tout au long du projet) et une justice distributive plus opérationnelle. Il s'agit là d'internaliser les coûts sociaux afin qu'ils ne soient plus supportés par les tiers mais par le producteur des impacts (protection contre le bruit, travail esthétique pour l'insertion paysagère de l'infrastructure, gestion partagée de la ressource piscicole).

Pour ce faire, encore faut-il que l'impact soit connu. Reconnaître un effet et son impact sur les individus ou la nature est parfois l'objet de tensions et d'incertitudes (comment évaluer les atteintes à la biodiversité ? comment matérialiser la gêne ressentie ? comment mettre en évidence des effets sur la santé ?). Aussi dans certains cas, le maître d'ouvrage participe-t-il au financement d'études (épidémiologiques, toxicologiques, environnementales, etc.) qui vont au-delà des exigences légales.

Si le maître d'ouvrage ne peut ni éviter, ni réduire les impacts provoqués par son projet, il lui reste l'alternative de la compensation. Elle exige de sortir d'une logique de monétarisation et d'entrer dans un principe de traitement concret -et souvent en nature- des maux. La compensation socioenvironnementale pourrait ainsi se définir comme l'attribution à une population subissant les effets négatifs d'une infrastructure d'un ensemble de mesures visant à rétablir un " équilibre " entre 
impacts négatifs et retombées positives : bourse du travail et accès privilégié aux emplois créés sur l'infrastructure aux "riverains"; requalification des quartiers et des réseaux particulièrement éprouvés par le fonctionnement de l'équipement; mise en place de fonds spécifiques pour les populations défavorisées riveraines; reconstitution de milieux naturel détruits. Ce type d'actions compensatoires, collectives, plus rarement individuelles, prend différentes formes aux Etats-Unis (Community Benefits Agreement) où elles peuvent s'adresser préférentiellement aux populations vulnérables afin de traiter des inégalités environnementales, au Canada (Ententes ou Impact and Benefits Agreement), dans une moindre mesure en France (e.g. les plans d'accompagnement de projet pour les lignes à haute tension).

Les porteurs de projet se voient ainsi contraints d'affiner leurs offres non pas pour acheter la paix sociale mais pour mieux intégrer leur infrastructure; non pas pour intervenir au seul niveau de l'insertion paysagère ou en prenant soin de quelques composantes naturelles, mais en respectant la structure sociale et environnementale du territoire.

\section{Glossaire}

Externalité : Impact positif ou négatif produit par l'activité d'un acteur dont il ne supporte pas les coûts mais qui influe sur le bien-être et le comportement d'acteurs tiers (pollution ou prise de valeur).

Inégalités environnementales: Impacts environnementaux pesant davantage et de manière disproportionnée sur des populations socio-économiquement fragiles et/ou appartenant à des minorités ethniques.

Ennoiement : Lors de la construction d'un barrage, immersion de surfaces boisées, agricoles et/ou résidentielles de plusieurs hectares pour constituer les bassins de rétention.

\section{Bibiographie}

Gobert J., 2012, «Les compensations territoriales: entre équité environnementale et justice sociale ? ", in Moreau S., Blanchon D., Gardin J. (dir), Justice et injustices environnementales, PUF, $230 \mathrm{p}$.

Simard L., 2006b, « Négocier l'action et l'utilité publiques. Les APC du transport de l'électricité en France et au Québec ", Négociations, n², p. $99-112$ 
